\title{
Role of cone beam computed tomography in dental diagnosis and treatment planning: An update
}

\author{
Abha Rani ${ }^{1, *}$, Varsha Kanjani ${ }^{2}$, Mamatha G.P ${ }^{3}$, Neetha M.C ${ }^{4}$, Rajeshwari G. Annigeri ${ }^{5}$ \\ ${ }^{1,2}$ Post Graduate Student, ${ }^{3,4}$ Professor, ${ }^{5} \mathrm{HOD}$, Dept. of Oral Medicine \& Radiology, College of Dental Sciences Davanagere, Karnataka, \\ India
}

*Corresponding Author: Abha Rani

Email: abha.rani.singh02@gmail.com

\begin{abstract}
Diagnostic treatment planning is crucial to achieve optimal success in oral rehabilitation, and this will be impossible without assistance of diagnostic imaging. For this reason, cone beam computed tomography (CBCT) was introduced and is known for its accuracy and variegated clinical importance. In current era, cone-beam tomography is aiding in the process of diagnosis by dispensing an exorbitant three dimensional image. CBCT not only be used in surgical fields, endodontics, prosthodontics, and orthodontics, for appropriate treatment planning and effective dental care, but also in forensics and radiotherapy. The clinical significance of this review is to update dental clinicians about CBCT applications in several fields of dental sciences.
\end{abstract}

Keywords: Cone beam computed tomography, Pathology, Radiology, Three-dimensional, Imaging.

\section{Introduction}

Oral radiology has been empowered by the imaging techniques which helped them to refurbish their diagnosis and subsequent treatment plans. Though, even in presence of number of advanced diagnostic techniques available, imaging has been considered as one of the most indispensable, accurate, precise and non-invasive technology for evaluation, analysis as well as deciding the current treatment plan. ${ }^{1,2}$

CBCT has several advantages that include:

1. Rapid Scan Time: A single rotation reduces the chances of appearance of artifacts related to the movement of the patient.

2. Beam Limitation: Availability of different field of view (FOV) results into limited irradiation and sharp image.

3. Image Accuracy: Image development with volume elements as declined as $0.076 \mathrm{~mm}$.

The reasons most commonly associated with artifacts production in CBCT includes X-ray beam artifact, subjective movement of patient and poor scanner detection. Image noise is an unnecessary additional pixel record due to considerate amount of scattering associated with the X-ray radiation. Another limitation of CBCT is inferior soft tissue contrast which is because of scattered radiation along with the image noise.

CBCT provides a 3-dimensional image viewing, enabling exact location and extent of lesions or any anatomical region. This is the reason why, CBCT isn't only used in surgical fields but also in fields of endodontics, orthodontics, periodontics and prosthodontics. But the excessive cost and considerable radiation dose have been responsible to confined CBCT use. $^{3}$

The source of ionizing radiation in CBCT gives a shape of a divergent pyramidal or cone shaped beam directed through the central $\mathrm{x}$-ray source to a X-ray detector placed opposite to the area of interest. Source of the X-rays and detectors placed opposite to each other rotates around a stationary fulcrum inside the region of interest. From a desired field of view (FOV), multiple sequential planar projection images of about 600 frames are obtained with a single complete rotation. FOV units of $<10 \mathrm{~cm}$ size (i.e. small FOV) are appropriate for dento-alveolar imaging and are hugely beneficial for endodontic applications, diagnosis of cysts and tumors. Medium FOV's ranging between 10 and $15 \mathrm{~cm}$ are useful for implant planning and pathological conditions in maxillomandibular region. Large FOV units ranging from 15 to $23 \mathrm{~cm}$ are indicated in the diagnosis of maxillofacial fractures, orthognathic surgery planning, orthodontic analysis and temporomandibular joint (TMJ) analysis. ${ }^{4,5}$

"Cone-beam computed tomography is moving from aphase of "as low as reasonably achievable-ALARA" to the phaseof "as low as diagnostically acceptable-ALADA." However, due to lacking experience about CBCT use in dentistry, unjustified prescription has increased exponentially in recent years. ${ }^{7-10}$ Keeping the fact in mind that the greater radiation dose is greater in CBCT than in comparison to conventional radiographs, it is must that the potential benefits of CBCT should outweigh the risks caused by the exposure to ionizing radiation exposure. ${ }^{11}$ Therefore prescription of CBCT should be justified appropriately and its usage for screening purposes alone should be strictly prohibited. The American Dental Association Council on Scientific Affairs Council has promoted CBCT operators to provide contribution to continuing education course, thus inorder to ensure a better understanding of dental personnel regarding radiation safety in the dental radiology setting. ${ }^{12}$

\section{Evolution}

Radiology has played extraordinary role in dental and medical diagnosis, treatment plan, and prognostic value of various diseases ever since the invention of X-rays in 1895 . Two-dimensional radiographs are the foreshadowed techniques in dentistry. These radiographic methods have certain limitations like magnification, distortions and 
superimpositions of structures restricting its diagnostic value. To overcome these limiting challenges 3D imaging techniques were introduced. Discovery by Sir Godfrey N. Hounsfield in 1972 of the leading commercial available computed tomography (CT) scanner. A newer generation of CT was developed in 1982 called cone beam CT (CBCT). The introduction of CBCT specifically dedicated to imaging the maxillofacial region lead to a true paradigm shift in imaging system. ${ }^{13,14}$ The first prototype of clinical CBCT scanner was originally devised as a cost-effective and efficient method for obtaining cross-sectional threedimensional images for radiotherapy and later (1982) for angiography. ${ }^{15}$ Columbia Scientific Inc., introduced 3D dental software in dentistry in 1988, and after 2 years, CBCT started to appear in dental research publications. ${ }^{16}$ First commercially available CBCT was introduced in Europe in 1998 and US in 2001. ${ }^{3}$

\section{Applications in Dentistry}

Oral Surgery: An amalgamation of low radiation dose and high-quality bony definition, has made CBCT alluring in dental imaging system for assessment the size, extent, and location of a tumor or cyst, its surrounding structures, and approximity to vital structures such as nerves and blood vessels as well as regions of bone destruction, bony deformities related to bisphosphonate-associated osteonecrosis of the jaw, bone grafts and accurate assessment of the involvement of maxillary sinus site and root configuration of impacted teeth and supernumerary in the head and neck, extracranial, paranasal, and temporal bone regions. ${ }^{17}$

Even the "spots" visible on conventional radiography can be pinpointed, eliminating the possibility of misinterpretation thus allowing the dentist to ameliorate their diagnosis. ${ }^{13,18,19}$

The accurate assessment of the inferior alveolar canal to the roots of mandibular third molar has reduced the injuries to nerve in extraction of impacted third molar drastically and also avoids loss of sensation to the lower lip during implant placement due to nerve injury. Although conventional 2D panoramic imaging provides clear image of the third molar when it is away from the canal but it is advisable to opt for 3D imaging approach in cases of radiographic superimposition of the structures..$^{20,21}$

CBCT has the ability to provide pinpoint details, so it becomes the technology of choice for midface fracture cases e.g., maxilla-mandibular fractures, orbital fracture management, interoperative visualization of the facial bones after fracture, and intraoperative observation during surgical procedures. $^{22-24}$

Cleft lip and palate evaluation and treatment planning is a tangled challenge for dentists. CBCT allows for the superior evaluation of dental age, arch segment positioning, and cleft size. Multislice CT and CBCT have been proved as reliable techniques in the volumetric assessment of bone defects in the alveolar and palatal regions. ${ }^{25}$

\section{Obstructive Sleep Apnea}

Obstructive sleep apnea is an anonymous and under diagnosed medical condition which is characterized by snoring and episodes of breathing cessation or the absence of respiratory airflow (10 seconds) during sleep, despite respiratory effort. ${ }^{26}$

CBCT, with its 3D presentation of the airway and its surrounding structures, provides better understanding of the intricacies. CBCT can evaluate the cross-sectional area, the volume, and the form, thus providing a more accurate anatomization than a $2 \mathrm{D}$ view, since it exemplifies the peculiarity that modifies airflow, which depicts the crosssectional area radius. ${ }^{27}$ This better vision of inward eye of both untreated obstruction cases and potential changes that can be made in the airway by treatment modality. To analyze changes in the upper airway with and without placement of a mandibular advancement device using different software have been used. Excellent segmentation was achieved, and made it possible to make airway minimum cross-sectional area and volumetric assessment. ${ }^{28}$

Inspite the fact that CBCT neither have the highest resolution like that of conventional $\mathrm{CT}$ and is nor the most sophisticated airway imaging method, this technology has been developing and used frequently, because of its lower radiation exposure dose. ${ }^{29}$

\section{Radiotherapy}

In recent years image guided radiation therapy (IGRT) has been stated to employ CBCT for patient positioning during radiation therapy. With $\mathrm{CBCT}$, a precise positioning of the patient i.e iso centre in radiotherapy machine is obtained with a single rotation immediately before delivering radiotherapy. It is further reconstructed in $<2 \mathrm{~min}$ for treatment planning. ${ }^{31}$

\section{Periodontics}

CBCT allows better figuring out of bone craters, crestal bone loss, fenestrations, and dehiscences and furcation involvements than digital intraoral radiography. The authors have also concluded that CBCT imaging is exclusivefor the detection of bone topography with only minimal error and lesion architecture but of similar accuracy as that of 2D for bone height evaluation. ${ }^{33}$ Most of the CBCT software includes tools for bone density evaluation, which aid to assess and predict the treatment modality, and its prognosis. $^{9}$

Despite of CBCT extraordinary usefulness in periodontics, it is not indicated as a routine method. But, CBCT is usually indicated in cases of infrabony (buccal or lingual) defects and furcation involvements, where conventional radiographic examinations do not provide the high resolution image needed for management. ${ }^{34,35} \mathrm{CBCT}$ can also useful within a period of 1 month for postoperative defect fill or bone density evaluation, which cannot be detected through normal radiographs, and it can also replace subtraction radiography. ${ }^{35}$ 
ST-CBCT is used for establishing minor bone defect size and early signs of periodontitis by computing periodontal ligament space, evaluating of gingival tissue, and the dimensions of the dentogingival unit, gingival margin and the facial bone crest, gingival margin and the cement-enamel junction (CEJ). ${ }^{36-38}$ It is also conducive for precise visualization and measurement of distances corresponding to the hard and soft tissue of the periodontium and dentogingival attachment apparatus.

It helps dental clinicians to evaluate post-surgical results of regenerative periodontal therapy. It also helps to select absorbable membrane with the optimal shape to fit into interproximal bony defect and decreases the time needed for GTR.

\section{Endodontics}

CBCT is more atheletic and finer to periapical radiographs in the depiction of periapical pathologies, signifying lesion intimacy to the maxillary sinus, or relative location to the mandibular canal. ${ }^{9} \mathrm{CBCT}$ provide better and precise anatomization for periradicular surgical procedures, periapical pathology demarcation, internal and external root resorption differentiation, and dentoalveolar trauma depiction.

CBCT can also be used for caries diagnosis inproximal and occlusal surface along with precise depth. However, its application in endodontic metallic restoration is limited as it produces metal artifacts thus reducing its diagnostic accuracy. ${ }^{35}$ CBCT imaging for caries should be limited to non-restored teeth. Sensitivity may increase with use of CBCT but it should not compromise specificity. ${ }^{35}$ CBCT can be used to differentiate solid from fluid-filled lesions (e.g., periapical granulomas from cysts) using grayscale values in the lesions. ${ }^{35} \mathrm{CBCT}$ plays an important role in endodontic therapy by identifying of all root canals (e.gsecond mesiobuccal canal (MB2) in maxillary first molars and aberrant pulpal anatomy, e.g., dens invaginatus) without any fail so that they can be accessed, cleaned, shaped, and obturated..$^{39,40}$

\section{Implantology}

CBCT provides cross-sectional images in three spatial planes (sagittal, axial, coronal) of the alveolar bone determining its height, width, density and angulations. Also, the loss of buccal or lingual cortical plates and unexpected concavities can also be demarcated on cross-sectional veiws, which aren't visible on panoramic images due to overlapping.CBCT images illustrate the quantity as well as quality of bone available for placement of implant as well as to demarcate the boundaries of significant vital structures in surgical preparation for dental implant planning for example incisive canal, mental foramen mandibular canal, and maxillary sinus. Cross-sectional images provide us knowledge about ridge patterns, such as irregular ridge, narrow crestal ridge, and knife shape ridge. ${ }^{10} \mathrm{CBCT}$ in implantology is indicated for determination of the distance of implant to vital anatomic structures to deciding if a bone graft or sinus lift is required or not by measuring alveolar bone thickness, density and bony contours, deciding the implant type and size for a particular patient and last but not the least for identification of the implant site and angulations ideal according to individual anatomization.

Computer-generated surgical guides (stereolithographic models) are being fabricated with the assistance of DICOM data i.e nothing but the CBCT image data to eliminate potential inaccuracy of impressions, conventional guide stents. The use of computer-guided implant surgery has really enhanced the dental implant team's ability to plan, place, and restore implants accurately with a level of precision. ${ }^{41}$

In peri-implantitis cases, the amount of bone present surrounding the implant can be assessed as radiolucency. ${ }^{42,43}$

\section{Temporomandibular Joint Assessment}

Due to complex anatomization and overlapping of structures in 2D imaging the diagnosis of TMJ disorders become very challenging. Though MRI is the gold standard for intra-articular components of the TMJ imaging, whereas conventional panoramic radiography is usually considered for routine evaluation of its bony components. ${ }^{44}$

But, conventional radiographs in two dimensions have low sensitivity, accuracy and reliability in evaluating the condylar region. CBCT high diagnostic quality images provides a complete radiographic evaluation of the bony components of the TMJ. ${ }^{45}$

Mandibular movement, function limitation, stiffness of the jaw, dysfunctioning related to trauma, pain, dysfunction, detecting condylar cortical erosion, cysts, visualization of soft tissue (ST), and fibro-osseous ankylosis can also be assessed by CBCT imaging. ${ }^{46}$ Due to significantly reduced radiation dose and cost compared to $\mathrm{CT}, \mathrm{CBCT}$ is becoming revolutionary investigational tool of choice for evaluating bony changes of the TMJ. ${ }^{44}$

CBCT can assess congenital/developmental malformations, malocclusion, degenerative diseases, ankylosis, joint remodelling following discectomy more precisely. The authors have concluded that CBCT accurately assess periarticular bony defects, osteophytes, sclerotic change and pathologic/degenerative changes, such as osteophytes, condylar erosion, condylar hyperplasia, and rheumatic disease. ${ }^{44,47,48}$

\section{Orthodontics}

Introduction of CBCT offered a 3D image that can be used to predict in orthodontic tooth movement in all three spatial planes. CBCT is an accurate tool for assessing the intimacy of impacted teeth to vital structures that could interfere with orthodontic movement. ${ }^{30}$

The angulation is well appreciated in CBCT 3D images, which would be difficult in conventional radiographs even when taken in two different (SLOB technique). It also helps in identification of any resorption of adjacent teeth (i.e., where positioning of maxillary canines are ectopic and incisor roots are suspected for resorption).

Structural and anatomic relations can be visualized accurately due to the cross-sectional imaging that nullifies 
the image overlapping. CBCT imaging has in recent past been largely explored in the field of orthodontics for evaluation of thickness of bone on the palatal aspect, skeletal growth patterns, age estimation by evaluating the dentition, upper airway assessment, and visualization of impacted teeth..$^{20,30}$

CBCT imaging allows a more widespread orthodontic diagnosis and more correct treatment planning by anatomization of impacted teeth precisely in 3D, treatment planning and growth assessments in orthognathic surgery cases, proper placement mini-implants as temporary anchorage devices (TADs), level of skeletal asymmetry and anomalies (cleft palate) assessment, ${ }^{49}$ as well as assessment of bone quality and dimensions and precise location of placement of mini -screw for anchorage to minimize complications. ${ }^{50}$

CBCT images are orthogonal images with a 1:1 measuring ratio i.e no magnification thus CBCT is considered as an accurate option than panoramic and other conventional 2D imaging.

Minute details of root resorption, bone width availability for the buccolingual movement of teeth, tooth inclination and torque can also be obtained from CBCT images. 3D cephalometry is beneficial in several ways including accurate measurement of horizontal and vertical growth, visual assessment of dentoskeletal relationships and facial esthetics. ${ }^{51,52}$

\section{CBCT in Forensic Odontology}

The clinical introduction of CBCT has created new opportunities in forensic odontology.Secondary dentine apposition is an age-related process that begins after tooth root get completely developed and continues throughout whole life. As age increases, secondary dentine lays on the walls of pulp cavity and decrease the size of pulp cavity. With this principle in mind, many attempts have been made to correlate the pulp cavity size and chronological age by the use of two-dimensional radiographic imaging techniques like panoramic or periapical radiographs for age estimation. ${ }^{53-54}$

Though the conventionally used periapical, lateral oblique, cephalometric, panoramic, digital imaging system displays only two dimensional images, the use of CT and CBCT have resulted in the dawn of three dimensional volumetric analysis as means of age assessment. Using CBCT acquisitions, multiple regression analysis including quantitative volumetric measurement of the tooth can be useful to determine significant variables for dental age estimation in living subjects. CBCT offers clear images of 3D volumetric anatomy of the pulp chamber and root canal by a single scan and at a lower radiation dose. CBCT evaluation of teeth is non-invasive technique requiring, neither tooth extraction nor sectioning, thus easy to accept on cultural, ethical, or scientific basis.

It provides an alternative to view the physiological as well as the pathological changes of the pulpo-dentinal complex in antemortem and postmortem scans. ${ }^{55}$ There is high scope of CBCT for victim identification and age estimation. CBCT is equally accurate in skeletal age assessment, especially when analyzing the cervical vertebra morphology. It also provides a 3D approach to the biologic aging by depicting images of the cervical spine for age assessment.

\section{Future Trends in CBCT}

The future of CBCT is the "ultra CBCT scanners" which is enhanced CT scanner. It provides specific information concerning the 3D structure of vascular channels, nerve supply of the region imaged, soft tissue and bone. ${ }^{30}$ Conventional cone CT imaging renders an 8-bit grayscale which yields 256 shades or 12-bit grayscale that provides 4,096 shades, whereas new ILUMA Ultra CBCT Scanner renders images in 14-bit grayscale, resulting in the shades of $16,384 . .^{30,57}$

The contrast-enhanced territories imaged by CBCT with selective intra-arterial contrast agent administration were employed to forecast allotment of microspheres. ${ }^{57}$

It also provide information about tumor and tissue perfusion presently which are unpredictable with digital subtraction angiography or $99 \mathrm{mTc}$ macro-aggregated albumin imaging, which should optimize 90Y (yttrium-90) microsphere delivery and reduce non-target embolization..$^{57,58}$

\section{Conclusion}

While clinical applications of CBCT have increased soar in current scenario, we must also bear its disadvantages and limitations into our mind before prescription. However, this should not demotivate dental surgeons from utilizing CBCT. But the judicious use of CBCT technology can outweigh inherent risks over its extraordinary potential benefits. Proper training and education in CBCT for oral maxillofacial radiologists and dentists is must to ensure judicious use of CBCT technology.

\section{Conflict of Interest: Nil.}

\section{References}

1. Ning R, Chen B. Cone beam volume CT mammographic imaging: feasibility study. In: Anto-nuk LE, Yaffe MJ, editors. Medical imaging 2001: physics of medical imaging proceedings of SPIE. vol. 4320. San Diego (CA): CA SPIE;2001. p. 655-664.

2. Feld-Kamp LA, Davis LC, Kress JW. Practical cone beam algorithm. J Opt Soc Am. 1984;A1(6):612-619.

3. Wisch-mann H-A et al. Correction of amplifier non-linearity, offset, gain, temporal artifacts, and defects for flat-panel digital imaging devices In: Anto-nuk LE, Yaffe MJ, editors. Medical imaging 2002: physics of medical imaging proceedings of SPIE. vol. 4682. San Diego (CA): CA SPIE; 2002. p. 427-437.

4. Pinsky HM, Dyda S, Pinsky RW, Misch KA, Sarment DP. Accuracy of three-dimensional measurements using cone-beam CT. Dentomaxillo fac Radiol. 2006;35:410-416.

5. Winter AA, Pollack AS, Frommer HH, Koenig L. Cone beam volumetric tomography vs. Medical CT scanners. N Y State Dent J. 2005;71:28-33.

6. Jaju PP, Jaju SP. Cone-beam computed tomography: time to move from ALARA to ALADA. Imaging Sci Dent. 2015;45:263-265 
7. Suomalainen A, Kiljunen T, Käser Y, Peltola J, Kortesniemi $\mathrm{M}$. Dosimetry and image quality of four dental cone beam computed tomography scanners compared with multislice computed tomography scanners. Dentomaxillofac Radiol. 2009;38:367-378.

8. Pauwels R, Beinsberger J, Collaert B, Theodorakou C, Rogers J, Walker A, et al. Effective dose range for dental cone beam computed tomography scanners. Eur J Radiol. 2012;81:267271.

9. Horner K, Islam M, Flygare L, Tsiklakis K, Whaites E. Basic principles for use of dental cone beam computed tomography: consensus guidelines of the European Academy of Dental and Maxillofacial Radiology. Dentomaxillofac Radiol. 2009;38:187-195.

10. Ludlow JB, Ivanovic M. Comparative dosimetry of dental CBCT devices and 64-slice CT for oral and maxillofacial radiology. Oral Surg Oral Med Oral Pathol Oral Radiol Endod. 2008;106:106-114.

11. The 2007 recommendations of the International Commission on Radiological Protection. ICRP Publication 103. Ann ICRP. 2007;37:1-332.

12. American Dental Association Council on Scientific Affairs. The use of cone-beam computed tomography in dentistry: an advisory statement from the American Dental Association Council on Scientific Affairs. J Am Dent Assoc. 2012;143:899902.

13. White SC, Pharoah MJ. The evolution and application of dental maxillofacial imaging modalities. Dent Clin North Am. 2008;52:689-705.

14. Scarfe WC, Farmen AG. What is cone beam CT and how does it work. DCNA. 2008;52:707-730.

15. Liguori C, Frauenfelder G, Massaroni C, Saccomandi P, Giurazza F, Pitocco F, et al. Emerging clinical applications of computed tomography. Med Devices (Auckl). 2015;8:265-278.

16. Orentlicher G, Goldsmith D, Abboud M. Computer-guided planning and placement of dental implants. Atlas Oral Maxillofac Surg Clin North Am. 2012;20:53-79.

17. Ogawa T, Enciso R, Shintaku WH, Clark GT. Evaluation of cross-section airway configuration of obstructive sleep apnea. Oral Surg Oral Med Oral Pathol Oral Radiol Endod. 2007;103:102-8.

18. Danforth RA, Peck J, Hall P. Cone beam volume tomography: An imaging option for diagnosis of complex mandibular third molar anatomical relationships. J Calif Dent Assoc. 2003;31:847-52.

19. Cervidanes LH, Bailey LJ, Tucker GR Jr, Styner MA, Mol A, Phillips CL, et al. Superimposition of 3D cone-beam CT models of orthognathic surgery patients. Dentomaxillofac Radiol. 2005;34:369-375.

20. Winter AA, Pollack AS, Frommer HH, Koenig L. Cone beam volumetric tomography vs. Medical CT scanners. N Y State Dent J. 2005;71:28-33.

21. Webber RL, Horton RA, Tyndall DA, Ludlow JB. Tunedaperture computed tomography (TACT). Theory and application for three-dimensional dento-alveolar imaging. Dentomaxillofac Radiol. 1997;26:53-62.

22. Araki M, Kameoka S, Matsumoto N, Komiyama K. Usefulness of cone beam computed tomography for odontogenicmyxoma. Dentomaxillofac Radiol. 2007;36:423-427.

23. Nair MK, Pettigrew JC, Jr, Mancuso AA. Intracranial aneurysm as an incidental finding. Dentomaxillofac Radiol. 2007;36:107-112.

24. Bianchi A, Muyldermans L, Di Martino M, Lancellotti L, Amadori S, Sarti A, et al. Facial soft tissue esthetic predictions: Validation in craniomaxillofacial surgery with cone beam computed tomography data. J Oral Maxillofac Surg. 2010;68:1471-1479.
25. Albuquerque MA, Gaia BF, Cavalcanti MG. Comparison between multislice and cone-beam computerized tomography in the volumetric assessment of cleft palate. Oral Surg Oral Med Oral Pathol Oral Radiol Endod. 2011;112(2):249-257.

26. McCrillis JM, Haskell J, Haskell BS, et al. Obstructive sleep apnea and the use of cone beam computed tomography in airway imaging: a review. Semin Orthod. 2009;15(1):63-69.

27. Ogawa T, Enciso R, Memon A, Mah JK, Clark GT. Evaluation of 3D airway imaging of obstructive sleep apnea with conebeam computed tomography. Stud Health Technol Inform. 2005;111:365-368.

28. McCrillis J, Farman A, Scarfe W, et al. Analysis of Airway Changes using CBCT with and without Placement of a Mandibular Advancement Device. Louisville, KY: University of Louisville School of Dentistry; 2008

29. Sharan A, Madjar D. Correlation between maxillary sinus floor topography and related root position of posterior teeth using panoramic and cross-sectional computed tomography imaging. Oral Surg Oral Med Oral Pathol Radiol Endod. 2006;102:375-381.

30. Heiland M, Schulze D, Blake F, Schmelzle R. Intraoperative imaging of zygomaticomaxillary complex fractures using a 3D C-arm system. Int J Oral Maxillofac Surg. 2005;34:369-375.

31. Scarfe WC, Farman AG. Cone beam computed tomography: A paradigm shift for clinical dentistry. Aust Dent Pract. 2007;18:102-110.

32. Drage NA, Brown JE. Cone beam computed sialography of sialoliths. Dentomaxillofac Radiol. 2009;38:301-305.

33. Mengel R, Candir M, Shiratori K, Flores-de-Jacoby L. Digital volume tomography in the diagnosis of periodontal defects: An in vitro study on native pig and human mandibles. $J$ Periodontol. 2005;76:665-673.

34. Dang V. Focus on cone beam computed tomography. Dent Pract. 2009;9:10-12.

35. Tyndall DA, Rathore S. Cone-beam CT diagnostic applications: Caries, periodontal bone assessment, and endodontic applications. Dent Clin North Am. 2008;52:825841.

36. Mohan R, Singh A, Gundappa M. Three-dimensional imaging in periodontal diagnosis-utilization of cone beam computed tomography. J Indian Soc Periodontol. 2011;15:11-17.

37. Afrashtehfar KI. Using bi-dimensional and tri-dimensional radiography in dentistry. Rev ADM. 2012;69:114-119.

38. Afrashtehfar KI, Cárdenas-Bahena JT, Afrashtehfar CD. Predictable immediate loading of mandibular implants. Tex Dent J. 2013;130:596-607.

39. Scarfe WC, Levin MD, Gane D, Farman AG. Use of cone beam computed tomography in endodontics. Int J Dent. 2009;2009:634567.

40. Patil S, Keshava Prasad BS, Shashikala K. Cone beam computed tomography: Adding three dimensions to endodontics. Int Dent Med J Adv Res. 2015;1:1-6.

41. Farman AG. Raising standards: Digital interoperability and DICOM. Oral Surg Oral Med Oral Pathol Oral Radiol Endod. 2005;99:525-526.

42. Uchida Y, Noguchi N, Goto M, Yamashita Y, Hanihara T, Takamori H, et al. Measurement of anterior loop length for the mandibular canal and diameter of the mandibular incisive canal to avoid nerve damage when installing endosseous implants in the interforaminal region: A second attempt introducing cone beam computed tomography. J Oral Maxillofac Surg. 2009;67:744-750.

43. Worthington P, Rubenstein J, Hatcher DC. The role of conebeam computed tomography in the planning and placement of implants. J Am Dent Assoc. 2010;141(Suppl 3):19S-24S.

44. Tsiklakis K, Syriopoulos K, Stamatakis HC. Radiographic examination of the temporomandibular joint using cone beam 
computed tomography. Dentomaxillofac Radiol. 2004;33(3):196-201.

45. Dahlström L, Lindvall AM. Assessment of temporomandibular joint disease by panoramic radiography: reliability and validity in relation to tomography. Dentomaxillofac Radiol. 1996;25(4):197-201.

46. de Boer EW, Dijkstra PU, Stegenga B, de Bont LG, Spijkervet FK. Value of cone-beam computed tomography in the process of diagnosis and management of disorders of the temporomandibular joint. Br J Oral Maxillofac Surg. 2014;52(3):241-246.

47. Alexiou K, Stamatakis H, Tsiklakis K. Evaluation of the severity of temporomandibular joint osteoarthritic changes related to age using cone beam computed tomography. Dentomaxillofac Radiol. 2009;38:141-147.

48. Honey OB, Scarfe WC, Hilgers MJ, Klueber K, Silveira AM, Haskell BS, et al. Accuracy of cone-beam computed tomography imaging of the temporomandibular joint: Comparisons with panoramic radiology and linear tomography. Am J Orthod Dentofacial Orthop. 2007;132:429-438.

49. Harrell WE Jr. Three-dimensional diagnosis \& treatment planning: The use of 3D facial imaging and 3D cone beam CT in orthodontics and dentistry. Aust Dent Pract. 2007;22:102113.

50. Bittencourt LP, Raymundo MV, Mucha JN. The optimal position for insertion of orthodontic miniscrews. Rev Odonto Cienc. 2011;26:133-138.

51. Periago DR, Scarfe WC, Moshiri M, Scheetz JP, Silveira AM, Farman AG. Linear accuracy and reliability of cone beam CT derived 3-dimensional images constructed using an orthodontic volumetric rendering program. Angle Orthod. 2008;78:387395.

52. Nakajima A, Sameshima GT, Arai Y, Homme Y, Shimizu N, Dougherty H Sr. Two and three-dimensional orthodontic imaging using limited cone-beam computed tomography. Angle Orthod. 2005;75:895-903.

53. Zaher JF, Fawzy IA, Habib SR, Ali MM. Age estimation from pulp/tooth area ratio in maxillary incisors among Egyptians using dental radiographic images. J Forensic Leg Med. 2011, 18(2):62-65.

54. Karkhanis S, Mack P, Franklin D. Age estimation standards for a Western Australian population using the coronal pulp cavity index. Forensic Sci Int. 2013;231(1-3):412 e1-412 e6.

55. Jaju PP, Jaju SP. Clinical utility of dental cone-beam computed tomography: Current perspectives. Clin Cosmet Investig Dent. 2014;6:29-43.

56. Scarfe WC, Farmen AG. What is cone beam CT and how does it work. DCNA. 2008;52:707-730.

57. Danforth RA, Peck J, Hall P. Cone beam volume tomography: An imaging option for diagnosis of complex mandibular third molar anatomical relationships. J Calif Dent Assoc. 2003;31:847-852.

58. Bulard RA. Ultra cone beam CT imaging the next generation of CBCT scanners. Eur J Radiol. 2009;99:461-468.

How to cite this article: Rani A, Kanjani V, Mamatha G.P, Neetha M.C, Annigeri R. G. Role of cone beam computed tomography in dental diagnosis and treatment planning: An update. 2018;4(4):122-117. 